\title{
An Update on Gender Parity Trends for Invited Speakers at Four Prominent Virology Conference Series
}

\author{
(D) Ann C. Palmenberg, ${ }^{a, b}$ (D) Robert F. Kalejta ${ }^{a, c}$ \\ alnstitute for Molecular Virology, University of Wisconsin-Madison, Madison, Wisconsin, USA \\ bDepartment of Biochemistry, University of Wisconsin-Madison, Madison, Wisconsin, USA \\ cMcArdle Laboratory for Cancer Research, University of Wisconsin-Madison, Madison, Wisconsin, USA
}

ABSTRACT Historically, men rather than women have been selected for invited speaking positions at the four prominent virology conference series we have followed since the 1980s. However, the low ratio of female representation in most earlier years (20\% in 1982 to 2010) has shown encouraging improvement $(37 \%$ in 2011 to 2017), particularly over the last few years (48\% in 2018 to 2020). Here, we describe this promising rise in inclusion and discuss factors that may help perpetuate and extend this overdue transformation toward gender parity.

KEYWORDS equity, gender, virology conference

D iscipline-specific research conferences are held to distribute new scientific information for the purposes of education and discussion. Invitations to present one's work at such conferences are professionally important and impact careers. Individual resumes highlight such invitations, which are taken as evidence of standing and achievement in one's field, and influence promotion packages, job applications, and grant submissions. In an earlier paper, we documented the decades-long dichotomy in invited talk selection between men and women for four prominent virology conferences, the annual American Society for Virology meeting (ASV), the annual International Herpesvirus Workshop (IHW), the triennial Positive-Strand RNA Virus Symposium (PSR), and the biennial Gordon Research Conference on Viruses and Cells (GRC) (1). For all four meeting series, the more historic speaker cohorts (1982 to 2010) uniformly showed much lower female representation (20\% average), than for the period (2011 to 2017) just prior to our previous report (37\% average). This report also discussed factors correlating with these apparent ratios, which for ASV, IHW, and GRC, involved the inclusion of at least one woman on the primary organization committee and, more importantly, for any invited conference roster, the participation of first-time speakers of any gender. Individual meetings that best met these criteria tended to have better gender parity. Here, we provide a follow-up review with extended observations on the promising recent rise in the inclusion of women as invited speakers. Encouragingly, the previous initial trend toward equity at these specific virology conferences has continued and even accelerated from 2018 to 2020 (to 48\% average representation).

Our tabulation methods are similar to those previously described (1). Invited speaker lists were obtained from online sources, entered into spreadsheets, and parsed by gender, year, and first/last name, as assigned by personal knowledge of individuals or published materials (e.g., websites). The most recent data augment previous rosters (1982 to 2017) with full descriptions of the following conferences: ASV 2018, 2019, and 2020; IHW 2018, 2019, and 2020; PSR 2019; and GRC 2019. Although neither the ASV nor the IHW talks were delivered in 2020 due to COVID-19 restrictions, we include those names because speaker selections for those intended conferences were decided and accepted many months prior to the meeting cancellations. The full data sets,
Citation Palmenberg AC, Kalejta RF. 2021. An update on gender parity trends for invited speakers at four prominent virology conference series. J Virol 95:e00224-21. https:// doi.org/10.1128/JVI.00224-21.

Editor Rozanne M. Sandri-Goldin, University of California, Irvine

Copyright $\odot 2021$ American Society for Microbiology. All Rights Reserved. Address correspondence to Ann C. Palmenberg, acpalmen@wisc.edu.

The views expressed in this article do not necessarily reflect the views of the journal or of ASM.

Accepted manuscript posted online 24 March 2021

Published 10 May 2021 

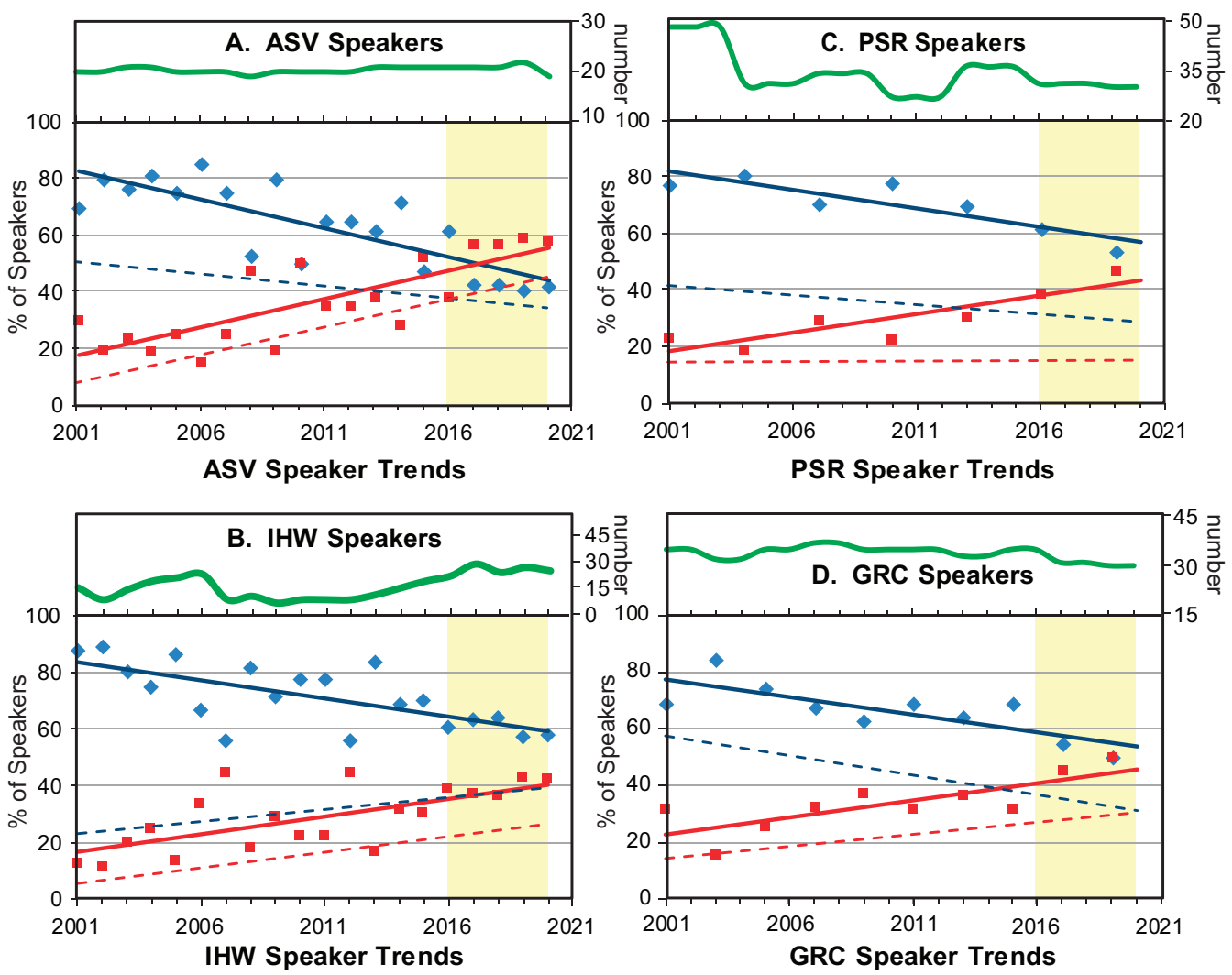

FIG 1 Time-dependent, gender-specific trends of the ASV, IHW, PSR, and GRC major invited speakers. Major speaker names were assembled, identified by gender, and plotted for the ASV (A), IHW (B), PSR (C), and GRC (D) series as described previously (1). The upper (green) lines track the absolute number of speakers per year. Male (blue diamonds) and female (red squares) contributors to each program are each shown as the percentage of total speakers for that year. Solid blue (male) and red (female) lines plot the linear regressions of these values. Parallel plots on the same scale (not shown) similarly recorded first-time male and female speakers. Linear regressions of these values (male, dotted blue lines; female, dotted red lines) are comparative. Yellow boxes highlight data for the last 5 years (2016 to 2020).

accurate to the best of our ability to vet names and gender, are publicly available online and are updated yearly (http://virology.wisc.edu/acp; select "Women in virology conference datasets").

Each conference meets at different intervals according to series customs and has speaker rosters that can differ in number from one iteration to the next. These four are certainly not the only international virology conferences, but are those for which we have complete data sets. As such, our findings may or may not be representative of other or all virology conferences. Although the cumulative records can reveal overarching trends, each of our conferences was tabulated individually. In this report, we focus on the years 2001 to 2020 , to unburden current times from the historic weight of the unchangeable past.

Figure 1 records the primary findings for each conference, plotted as before (1), but only for the years 2001 to 2020 . The blue and red symbols in each panel record the proportions of male and female major speakers in a given year. The solid regression lines are necessarily reciprocal and are calculated here based on data since 2001. Each graph has an uppermost green line with the actual number of included speakers. In parallel plots (data not shown), we separated out new names, male or female, which appeared for the first time in any given year, relative to full rosters from the preceding history of each series. The regression trends since 2001 for first-time speakers (dotted lines) are superimposed on the Fig. 1 graphs. The larger the vertical distance between any solid line and its same-color dotted line, the higher the proportion of historic reinvited names relative to new ones. Yellow boxes highlight data for the past 5 years, which, as stated above for ASV and IHW, include the finalized 2020 rosters. 
TABLE 1 Women as percentages of invited speakers

\begin{tabular}{lllll}
\hline & \multicolumn{4}{c}{ \% of invited female speakers (no. of meetings) for: } \\
\cline { 2 - 5 } Interval & ASV & IHW & GRC & PSR \\
\hline $2000-2004$ & $24(5)$ & $17(5)$ & $24(2)$ & $21(2)$ \\
$2005-2009$ & $26(5)$ & $28(5)$ & $32(3)$ & $29(1)$ \\
$2010-2014$ & $37(5)$ & $27(5)$ & $34(2)$ & $26(2)$ \\
$2015-2020$ & $54(6)$ & $38(6)$ & $42(3)$ & $43(2)$ \\
\hline
\end{tabular}

In the four Fig. 1 panels, ASV (Fig. 1A), IHW (Fig. 1B), PSR (Fig. 1C), and GRC (Fig. 1D), the blue solid lines point down and the red solid lines point up, meaning that each series has been including proportionally more women during the past 2 decades. It is of particular interest, within the yellow boxes indicating just the last 5 years, that for 3 of these 4 conferences, there was at least 1 year when the female rosters almost met, or in some cases exceeded, those of men. To better capture the time-dependent improvement across all series, Table 1 bins the same information in 5-year increments. All of these conferences show clear gains in female representation, with marked acceleration especially in the last 5 years.

Our previous analyses made it clear that including more first-time speakers correlated with increased female representation. From the full data set, we summed the increase in new names added cumulatively to all four rosters since 1982 (data not shown). Figure 2A graphs those records from 2001 to 2020. The slope differential for the male (blue) and female (red) lines means that on average new men have been added twice as fast as new women over the past 2 decades. As with the historic pools from earlier decades, this means that men are still being augmented faster than
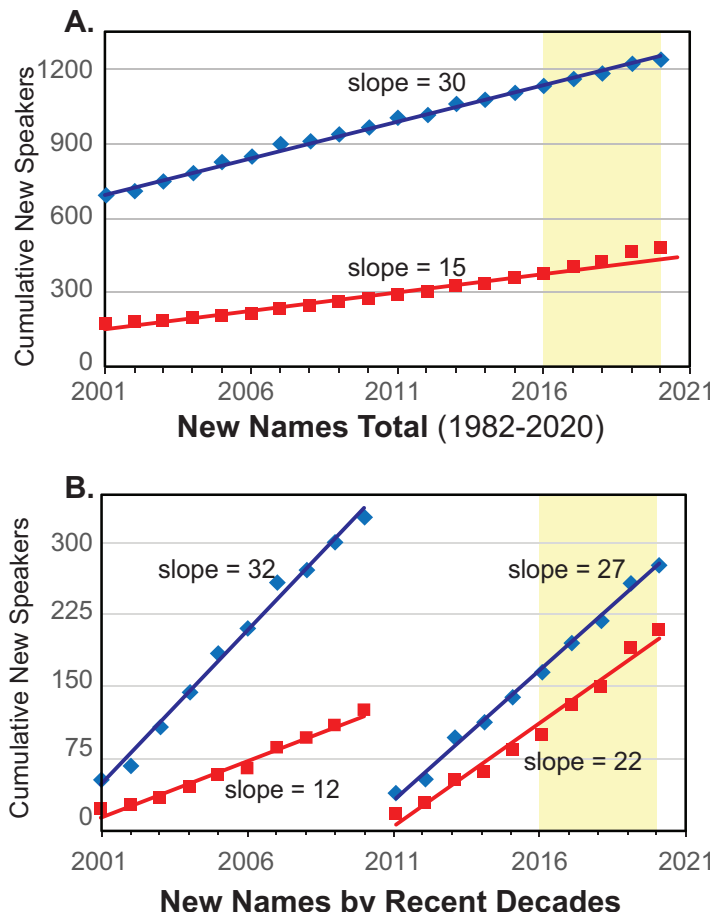

FIG 2 Cumulative new speaker addition. (A) The cumulative summation of new names (male, blue diamonds; female; red squares) added to ASV, IHW, PSR, or GRC major speaker rosters from 1982 to 2020 are plotted as a function of year. Linear regressions of these values from 2001 to 2020 (solid lines) were used to calculate slopes. (B) Similar to the analysis in panel A, but discarding previous history; the inclusion trends are considered independently for each of the most recent decades, 2001 to 2010 and 2011 to 2020 . All $R^{2}$ values are $>0.980$. Yellow boxes highlight data for the last 5 years (2016 to 2020). 
TABLE 2 New names (female/male) as percentages of invited speakers

\begin{tabular}{lllll}
\hline & \multicolumn{4}{c}{ \% of new invited speakers (female, male) at: } \\
\cline { 2 - 5 } Interval & ASV & IHW & GRC & PSR \\
\hline $2000-2004$ & 15,44 & 8,28 & 11,36 & 16,39 \\
$2005-2009$ & 13,50 & 14,27 & 12,29 & 18,41 \\
$2010-2014$ & 30,44 & 15,35 & 13,30 & 18,44 \\
$2015-2020$ & 42,34 & 26,38 & 17,20 & 18,27 \\
\hline
\end{tabular}

women, a contributing factor as to why generic speaker recycling tends to decrease parity. But when the newest data are split by decade (Fig. 2B), it becomes evident that the last 10 years document a strong overall increase in the addition of new women, although the rate and numbers still lag behind those of new men. Table 2 parses the binned information for each conference by 5 -year intervals. While in total, the proportion of new male speakers still exceeds that of new women, the percentage of all new speakers for most conferences is increasing. This is especially evident for the IHW, where both dotted lines (Fig. 1B) display positive slopes. Continued monitoring of such proactive addition rates could help prevent a return to the habitual recycling of speakers that in the past impeded progress across all conferences. Our data reemphasize that the introduction of first-time speakers, both female and male, remains an important step forward toward equity and inclusivity.

We hope that the awareness provided by analyses such as these, along with continued dialog and increased vigilance, will sustain and enhance the recent positive trends. Still, it remains necessary to bolster retrospective analyses with prospective measures. Several scientific conference series (e.g., Keystone, GRC, and ASV) now have published codes of conduct for attendees that forbid harassment or inappropriate behavior. However, few of these have yet agreed upon publicly defined criteria for their meetings' speaker selection. Proactive statements of intention should never contain numerical benchmarks (quotas), but should guide organizers through factors such as equity and inclusivity, in addition to scientific quality and topic timeliness factors, that they should consider when selecting speakers. Any conference hierarchy could demonstrate their level of commitment to such principles by publishing their expectations for speaker selection criteria, thus ensuring that both organizers and attendees are aware of current mores. Setting such precedents would hopefully catalyze the empowerment of populations marginalized because of their gender, race, national origin, sexual orientation, or other identities, to continue to force change toward equity and inclusivity.

Finally, we remind readers that the visibility provided by selection as an invited speaker at a conference is seen not only by manuscript reviewers and editors, study section members, and tenure committees, but also by the postdocs and trainees (both women and men) in the audiences. Today's leaders must actively promote and exemplify equity, diversity, and inclusivity to engrain these concepts into the hearts and minds of the next generation of leaders. To do so would maintain, or preferably extend, the recent advances we have reported. Tomorrow's scientists are watching. Let's show them who we want to be.

\section{ACKNOWLEDGMENTS}

We thank Donna Neumann for helpful comments on the manuscript.

A.C.P. analyzed the data. A.C.P. and R.F.K. wrote the text.

\section{REFERENCE}

1. Kalejta RF, Palmenberg AC. 2017. Gender parity trends for invited speakers at four prominent virology conference series. J Virol 91:e00739-17. https:// doi.org/10.1128/JVI.00739-17. 
